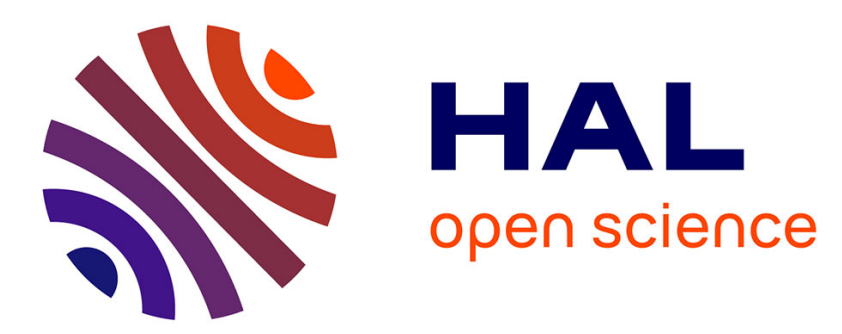

\title{
Les sources de distraction au volant et leur dangerosité : Enquête auprès des membres de la communauté universitaire de l'Université Saint-Joseph de Beyrouth
}

\author{
Ramzi Salamé
}

\section{- To cite this version:}

Ramzi Salamé. Les sources de distraction au volant et leur dangerosité : Enquête auprès des membres de la communauté universitaire de l'Université Saint-Joseph de Beyrouth. RTS - Recherche Transports Sécurité, 2016, Distractions au volant, 2015 (03-04), pp.199-210. 10.4074/S0761898016004064 . hal01670602

\section{HAL Id: hal-01670602 \\ https://hal.science/hal-01670602}

Submitted on 21 Dec 2017

HAL is a multi-disciplinary open access archive for the deposit and dissemination of scientific research documents, whether they are published or not. The documents may come from teaching and research institutions in France or abroad, or from public or private research centers.
L'archive ouverte pluridisciplinaire HAL, est destinée au dépôt et à la diffusion de documents scientifiques de niveau recherche, publiés ou non, émanant des établissements d'enseignement et de recherche français ou étrangers, des laboratoires publics ou privés. 


\title{
Les sources de distraction au volant et leur dangerosité : Enquête auprès des membres de la communauté universitaire de l'Université Saint-Joseph de Beyrouth
}

\author{
Sources of Driving Distraction and their Dangerousness: \\ A Survey among the Members of University Community of Saint-Joseph University in Beirut
}

\author{
Ramzi Salamé \\ (C) IFSTTAR et Éditions NecPlus 2015
}

Résumé Cette recherche a exploré les principales sources de distraction au volant chez les membres d'une communauté universitaire du Liban en termes d'incidence de ces sources de distraction sur les collisions, de perception de la dangerosité de chacune d'elles et d'occurrence des comportements les concernant. Les résultats d'une enquête en ligne obtenus auprès de 605 personnes démontrent que $30 \%$ des répondants ont eu au moins une collision due à la distraction au volant avec prépondérance, comme sources de distraction, du fait d' « être absorbé par ses propres pensées » et l'utilisation du téléphone portable pour parler, lire ou écrire un message. L'occurrence des comportements de distraction s'avère être en corrélation négative avec la perception de la dangerosité. Cependant, l'utilisation du téléphone portable et certaines autres sources apparentées de distraction s'avèrent moins obéir à l'impératif du danger qui leur est assigné et reflètent la difficulté des personnes de résister à la tentation d'une telle utilisation malgré le danger perçu. Cette recherche révèle aussi, chez la population étudiée, certaines différences démographiques quant à l'accidentalité due aux diverses sources de distraction.

Mots clés distraction · conduite automobile $\cdot$ universitaires - sources · dangerosité · téléphone portable

\footnotetext{
Abstract This study has explored the main sources of distracted driving among members of university community in Lebanon in terms of incidence of these sources on crashes, perception of level of dangerousness of each of them, and occurrence of the related behaviors. Results of

Ramzi Salamé $(\bowtie)$

Titulaire de la Chaire Fondation Renault - Université Saint-Joseph en Management de la sécurité routière Campus de l'Innovation et du Sport, Rue de Damas, Beyrouth, Liban

e-mail : ramzi.salame@usj.edu.lb
}

an online survey provided by 605 persons show that $30 \%$ of respondents have had at least a crash due to distracted driving with preponderance, as sources of distraction, of "being absorbed by own's thoughts" and the use of mobile phone to have a conversation, read or write a message. The occurrence of behaviors related to the sources of distraction appears to be negatively correlated with the perception of dangers of these same sources. However, the use of the mobile phone and certain other comparable sources of distraction appear to be less influenced by the perceived level of dangerousness and reflect the difficulty of the subjects to resist to the temptation of such use despite the danger associated to them. This study also reveals certain demographic differences among the concerned population as regards the occurrence of crashes related to the various sources of distraction.

Keywords Distraction · Driving · University · Sources · Dangerousness $\cdot$ Mobile phone

\section{Introduction}

Il est communément admis, autant dans la communauté scientifique que parmi les professionnels de la sécurité routière que la distraction au volant constitue un comportement dangereux autant pour les conducteurs de véhicules que pour leurs passagers et les autres usagers de la route. Cependant, ce n'est que récemment qu'il y a eu, parmi les théoriciens et les praticiens de la sécurité routière, un consensus suffisant sur la définition exacte du concept de distraction au volant et sur sa différentiation des autres formes d'inattention pendant la conduite de véhicules motorisés $[1,2,3,4]$.

Ainsi, les auteurs décrivent quatre formes principales de défauts de l'attention, soit : l'attention restreinte du conducteur due, en particulier, à des facteurs biologiques, l'attention mal dirigée due à une erreur dans la priorisation 
de l'attention, l'attention négligente ne fournissant pas suffisamment d'efforts pour saisir adéquatement la totalité de la situation de conduite et l'attention hâtive se contentant d'une saisie superficielle de la situation de conduite $[3,4]$.

De même, dans l'ouvrage collectif édité par Chapon, Garaude et Fort [5], trois sources principales de défauts d'attention au volant sont décrites : la distraction liée à la multi-activité au volant, l'inattention résultant $\mathrm{du}$ vagabondage de la pensée du conducteur et les troubles spécifiques de la sélectivité de l'attention dus, entre autres, au vieillissement, à certaines pathologies, aux addictions et à la prise de médicaments.

À la différence des quatre formes d'inattention susmentionnées, Engstrom et al. [2] proposent de définir la distraction au volant comme étant «le détournement de l'attention d'activités cruciales pour une conduite sécuritaire vers une activité rivale » $[2 ;$ p. 5], et Regan et Strayer [3] d'ajouter « qui peut résulter en l'absence d'attention ou en une attention insuffisante aux activités cruciales pour une conduite sécuritaire » [p. 5].

Dans leur taxonomie des comportements d'inattention au volant, Regan et al. [4] et Regan et Strayer [3] mentionnent que les activités rivales qui peuvent mener à la distraction au volant peuvent être internes à l'esprit de la personne (tels les rêves éveillés), internes au véhicule (et ils donnent comme exemple, parler au téléphone portable ou se préoccuper outre mesure des indications du tableau de bord) ou extérieurs au véhicule (tel lire un panneau publicitaire). Ces auteurs, ainsi que d'autres auteurs cités par Engstrom et al. [2] différencient aussi les sources de distraction reliées à la tâche de conduite et les sources de distraction non reliées à cette tâche.

De même, Fort [1] différencie les distractions externes au véhicule des distractions internes au véhicule qu'elle classe en deux catégories. La première catégorie concerne les distractions physiques ou motrices qui impliquent l'usage des mains à d'autres tâches que la conduite et, "dans la majorité des cas », le détournement de la vue de la route, et qui peuvent être reliées ou non à la manipulation des systèmes de contrôle du véhicule. La deuxième catégorie concerne les distractions cognitives qui incluent l'utilisation du téléphone portable et d'autres systèmes d'information.

Par ailleurs, des recherches récentes sur la distraction au volant $[6,7]$ ont ajouté à ce qui précède comme sources de distraction, entre autres, la gestion de l'interaction avec les autres passagers du véhicule et la gestion d'événements survenant à l'intérieur de celui-ci.

L'objectif de cette étude était de saisir l'ampleur du phénomène de distraction au volant dans la communauté universitaire de l'Université Saint-Joseph de Beyrouth, et de ses répercussions en termes de sécurité routière et d'étudier les paramètres qui y sont associés en vue de développer, si nécessaire, des contre-mesures et une politique institutionnelle à ce sujet, en particulier pour contrer le phénomène d'utilisation du téléphone portable pendant la conduite automobile chez les membres de la communauté universitaire.

Compte tenu de l'absence complète d'études sur la sécurité routière au Liban et, plus particulièrement, sur la distraction au volant, il n'était pas possible de formuler des hypothèses spécifiques à ce sujet. L'observation aléatoire des comportements des conducteurs de véhicules automobiles permettait cependant de croire que l'utilisation du téléphone portable au volant était relativement répandue dans la population libanaise, sans pour autant savoir si cela provoquait des collisions et quelles en étaient les conséquences.

\section{Méthodologie}

\subsection{Instrument utilisé}

En vue d'effectuer cette étude, un questionnaire à l'intention des membres de la communauté universitaire (enseignants, employés et collaborateurs de l'Université) a été élaboré en s'inspirant des études antérieures sur ce sujet, notamment celles de la Canadian Automobile Association [8], de la Société de l'assurance automobile du Québec [9] et de la National Highway Transport Safety Administration/NHTSA $[6,7]$.

Le pivot de ce questionnaire consiste dans 26 items qui représentent autant de sources de distraction au volant réparties comme suit :

a. sources de distraction relatives à la gestion du véhicule et de ses équipements (4 items) ;

b. sources de distraction relatives à la gestion d'événements à l'intérieur du véhicule non reliés à la tâche de conduite (12 items) dont 2 items concernent l'écoute de la musique et la manipulation de la radio, 3 items concernent la gestion de l'interaction avec les autres passagers, 3 items concernent les comportements de manger et boire, fumer et s'adonner à des soins personnels, 2 items concernent le contrôle d'objets, d'animaux ou de personnes et 2 concernent la lecture de matériel écrit ou l'écriture, hormis ce qui concerne l'utilisation du téléphone portable pour de tels comportements ;

c. sources de distraction externes au véhicule ( 3 items) ;

d. sources de distraction relatives à l'utilisation du téléphone portable durant la conduite (6 items) ;

e. sources de distraction internes à l'esprit du conducteur (1 item).

Il était demandé aux sujets de fournir des réponses à ce qui suit : 
1. Leur perception de la dangerosité de chacun des 26 items sur une échelle Likert en quatre points libellés comme suit : «Pas dangereux du tout » $(\operatorname{cote} 0) ;$ «Peu dangereux » (cote 1$)$; «Assez dangereux » (cote 2$)$; « Très dangereux » (cote 3$)$.

2. Un auto-rapport sur la fréquence d'occurrence de chacun des 26 comportements de distraction chez le répondant échelonnée comme suit : « Rarement/Jamais ou presque » $($ cote 0$) ;$ « Parfois » $($ cote 1$) ;$ « Souvent » $(\operatorname{cote} 2) ;$ « De façon » régulière (cote 3$)$.

3. Un auto-rapport sur l'occurrence de collisions dues à la distraction au volant, la source de distraction concernée et les conséquences des collisions en termes de dégâts matériels mineurs, dégâts matériels majeurs, blessés légers, blessés graves et décès.

4. Des données sociodémographiques, y compris la possession de permis de conduire et l'utilisation d'une voiture privée.

\subsection{Méthode d'investigation}

Le questionnaire en version électronique fut posté sur le site interne des enquêtes de l'Université et un courriel fut envoyé à l'ensemble de la population visée, soit tous les membres du personnel enseignant et du personnel administratif, avec indication du lien vers l'enquête en ligne et une invitation à répondre au questionnaire. Un seul rappel a été envoyé aux mêmes personnes trois semaines après la première invitation à répondre.

\subsection{Caractéristiques des répondants}

Compte tenu de la grande variété des profils des membres de la communauté universitaire visés, il n'est pas possible d'établir le degré de représentativité de l'échantillon ainsi obtenu. En effet, la communauté universitaire est composée de personnes de divers niveaux d'éducation et dont un grand nombre, selon les sources de l'administration de l'Université, n'utilise pas nécessairement le courriel comme moyen de communication. Aussi, force est de constater que si 893 personnes ont accédé au questionnaire sur une population de plus de 2000, 260 personnes n'ont fourni des réponses qu'à la partie concernant les données démographiques et 28 autres personnes ont fourni des réponses à cette partie et à la question concernant la perception de dangerosité des divers comportements inclus dans le questionnaire. Ces sujets n'ont donc pas été inclus dans les analyses statistiques qui suivent, de sorte que celles-ci ne concernent que les 605 personnes qui ont répondu à la question concernant l'occurrence de collisions dues à la distraction au volant dans leur cas personnel.
Compte tenu de ce qui précède, il convient de considérer cet échantillon comme population en soi avec ses propres caractéristiques.

Le tableau 1 fournit les données concernant les caractéristiques sociodémographiques des répondants qui ont fourni des réponses valides eu égard à l'occurrence de collisions dues à la distraction, en termes d'âge, d'expérience de conduite automobile, de statut matrimonial, de diplôme et de l'estimation des kilomètres parcourus par année.

Il apparaît de ce tableau que $29,8 \%$ des répondants (180/605) rapportent avoir déjà eu une collision due à la distraction au volant, avec une légère prépondérance de ce fait chez les hommes $(34,1 \%$ des répondants masculins $(84 / 246)$ versus $26,7 \%$ des répondantes $(96 / 359))$. D'après le test de chi carré, cette différence est significative et dénote que les hommes ont tendance à avoir eu plus de collisions que les femmes $(\mathrm{X} 2=3,841 ; \mathrm{dl}=1 ; \mathrm{p}=0,05)$. Cependant, compte tenu que les femmes sont significativement plus jeunes que les hommes et ont vraisemblablement parcouru moins de kilomètres alors qu'elles sont au volant, à la fois parmi les personnes qui ont eu des collisions dues à la distraction que parmi celles qui n'en ont pas eu, il est possible de croire que l'avantage des femmes à ce propos est dû à la différence de kilomètres parcourus plutôt qu'à une différence de genre.

Les données du tableau 1 révèlent par ailleurs ce qui suit :

1. Les deux groupes de femmes sont plus jeunes que les deux groupes d'hommes et ont un nombre inférieur d'années depuis l'obtention du permis de conduire, alors qu'autant les deux groupes d'hommes que les deux groupes de femmes ne se différencient pas entre eux sur ces deux variables.

2. La grande majorité des répondants ont déjà obtenu un diplôme de deuxième ou de troisième cycle et ceci est particulièrement vrai chez les hommes $(77,4 \%)$ qui rapportent avoir déjà eu des collisions dues à la distraction au volant $(65 / 84)$ et chez les femmes $(71,9 \%)$ qui rapportent n'en avoir pas déjà eu (189/263), les autres répondants étant aussi universitaires à de très rares exceptions. Ceci s'explique par la prépondérance, parmi les répondants, d'enseignants de l'Université et de cadres et employés professionnels.

3. Les personnes célibataires sont majoritaires parmi les femmes, alors que le statut marital ne différencie pas les personnes en termes d'occurrence de collisions dues à la distraction au volant.

4. Le nombre estimé de kilomètres parcourus au cours des douze derniers mois est généralement semblable d'un groupe à l'autre et correspond aux estimations nationales dans ce domaine, sauf pour le groupe de femmes qui rapportent n'avoir pas déjà eu de collisions dues à la distraction au volant où l'estimation du nombre de 


\begin{tabular}{|c|c|c|c|c|}
\hline \multirow[b]{2}{*}{ Variable } & \multicolumn{2}{|c|}{$\begin{array}{l}\text { Répondants } \\
\text { ayant eu des collisions }\end{array}$} & \multicolumn{2}{|c|}{$\begin{array}{c}\text { Répondants } \\
\text { n'ayant pas eu des collisions }\end{array}$} \\
\hline & $\mathbf{M}$ & $\mathbf{F}$ & $\mathbf{M}$ & $\mathbf{F}$ \\
\hline Nombre & 84 & 96 & 162 & 263 \\
\hline Moyennes et écarts-types de l'âge & $42,4(14,4)$ & $34,6(12,5)$ & $43,8(15,0)$ & $36(11,9)$ \\
\hline $\begin{array}{l}\text { Moyennes et écarts-types du nombre } \\
\text { d'années depuis l'obtention du } \\
\text { permis de conduire }\end{array}$ & $23,3(13,7)$ & $14,8(10,9)$ & $25,0(14,9)$ & $15,8(11,1)$ \\
\hline $\begin{array}{l}\text { Pourcentages ayant des diplômes } \\
\text { universitaires de } 2^{\mathrm{e}} \text { ou } 3^{\mathrm{e}} \text { cycle }\end{array}$ & 77,4 & 63,5 & 54,3 & 71,9 \\
\hline Pourcentages de célibataires & 34,1 & 57,9 & 34,4 & 54,5 \\
\hline $\begin{array}{l}\text { Moyenne des kilomètres parcourus au } \\
\text { cours des douze derniers mois }\end{array}$ & 14875 & 13600 & 14600 & 11500 \\
\hline
\end{tabular}

kilomètres parcourus est légèrement inférieure à celles concernant les trois autres groupes.

\section{Résultats}

\subsection{Sources de distraction ayant causé une collision}

Le tableau 2 présente les sources de distraction ayant causé des collisions telles qu'évoquées par les répondants selon le genre de ceux-ci. Il est à noter que 28,1\% des hommes et $28,6 \%$ des femmes n'ont pas indiqué la source de distraction qui était en cause lors de la collision.

Il apparaît clairement de ce tableau que les deux sources principales de distraction au volant ayant causé des collisions relèvent du fait d'être absorbé par ses propres pensées et de l'utilisation du téléphone portable. En effet, chacune de ces sources est évoquée dans $29,5 \%$ des cas. La gestion d'une situation à l'intérieur du véhicule vient en troisième place avec 13,2 \% des cas, suivie par la distraction par le paysage ou par un événement extérieur au véhicule $(7,8 \%$ des cas) et par la musique trop forte et la manipulation de la radio ( $7 \%$ des cas). Il est ainsi utile de mentionner que parmi les items relatifs à la manipulation des équipements du véhicule, seule la manipulation de la radio et de l'équipement de musique est mentionnée comme source de distraction ayant causé une collision.

Les autres 13,1\% des cas sont partagés entre d'autres sources de distraction impliquant toujours un comportement proactif du conducteur du véhicule à l'intérieur de celui-ci et ne concernent aucun des items relatifs à la gestion des équipements du véhicule indiquant ainsi la maîtrise de celleci par les répondants.

Il faudrait cependant noter que, hormis le fait d'être absorbé par ses propres pensées, l'utilisation du téléphone portable et le fait de boire ou manger où les deux genres ont des occurrences similaires de collisions, ceux-ci ont tendance à se distinguer l'un de l'autre sur les cinq autres variables (X2 calculé $=9,03$, comparé à X2 théorique de $9,48$, pour $\mathrm{dl}=4, \mathrm{p}=0,05)$. Ainsi les femmes ont tendance à être davantage affectées par la gestion des situations à l'intérieur du véhicule et par les comportements reliés à la musique et à la radio, alors que les hommes ont tendance à être davantage affectés par la distraction relative aux paysages extérieurs et au fait d'avoir une conversation avec les autres passagers du véhicule, les femmes évoquant, par ailleurs, d'autres causes de baisse de l'attention ayant trait à des déficits de la vigilance causés par le sommeil, la fatigue et la prise d'alcool. Compte tenu du nombre restreint de répondants, ces différences doivent être considérées comme des tendances à être corroborées ou infirmées par d'autres recherches plus étendues.

\section{2. Âge des répondants ayant eu une collision selon la source de distraction et le genre}

Le tableau 3 présente la moyenne de l'âge des répondants ayant eu des collisions selon la source de distraction et le genre. Il présente aussi le pourcentage de répondants ayant 26 ans ou moins pour chacune des sources de distraction ayant causé une collision. Le choix de cette limite d'âge 
Tableau 2 Sources de distraction ayant causé des collisions selon le genre

\begin{tabular}{|c|c|c|c|}
\hline & M (\%) & F (\%) & Total $(\%)$ \\
\hline 1. Être absorbé par ses propres pensées & 28,3 & 30,4 & 29,5 \\
\hline 2. Utilisation du téléphone portable pour parler, lire ou écrire des messages & 30 & 29 & 29,5 \\
\hline 3. Gérer une situation à l'intérieur du véhicule & 10 & 15,9 & 13,2 \\
\hline 4. Être distrait par le paysage ou un événement extérieur au véhicule & 11,7 & 4,3 & 7,8 \\
\hline 5. Être distrait par la musique trop forte ou par la manipulation de dispositifs d'écoute de la musique & 5 & 8,7 & 7 \\
\hline 6. Parler à un passager du véhicule & 8,3 & 2,9 & 5,4 \\
\hline 7. Manger et boire, avoir une activité liée au tabac & 5 & 4,3 & 4,7 \\
\hline 8. Diverses autres sources de distraction & 1,7 & 4,5 & 3 \\
\hline Total des répondants ayant indiqué la source de distraction & 60 & 69 & 129 \\
\hline
\end{tabular}

se justifie par le fait que les recherches récentes sur la maturation du cerveau considèrent cet âge comme âge charnière d'achèvement de la maturité biologique du lobe préfrontal, siège de la pensée raisonnée, de la planification et de l'anticipation, ainsi que du contrôle de la prise de risque et des inclinations à agir de façon spontanée ou irréfléchie [10].

Les données du tableau 3 montrent que la moyenne d'âge des répondants masculins ressemble à celle de l'ensemble des répondants quand il s'agit des sources de distraction ayant causé des collisions relatives au fait d'être absorbé par ses propres pensées, de parler au téléphone portable et de manger ou boire, alors que la moyenne d'âge est, pour ce groupe de répondants, plus élevée lorsqu'il s'agit de gérer une situation à l'intérieur du véhicule. Par contre, les moyennes d'âge sont plus basses de façon notable lorsqu'il s'agit des collisions provoquées par la distraction due à la lecture ou l'écriture d'un message texte, la contemplation du paysage extérieur, la musique trop forte et le fait de parler à un passager. La domination nette des moins de 26 ans ne se vérifie cependant qu'en ce qui concerne le danger de parler à un passager du véhicule.

Par contre, même si pour les sujets féminins le profil d'âge concernant les diverses sources de distraction ressemble sur certains points à celui de l'ensemble des répondants, il en diffère de façon notable sur trois points, soit écrire un message texte, écouter une musique trop forte et parler à un passager du véhicule où l'âge est plus bas. Par ailleurs, il y a, parmi les répondantes ayant eu des collisions, une nette prédominance des moins de 26 ans (pourcentage allant de $33 \%$ à $63 \%$ ) par rapport aux répondantes n'ayant pas eu de collision (28,5\%). Cependant, à nouveau, compte tenu du nombre restreint de répondants, ces différences doivent être considérées comme des tendances à être corroborées ou infirmées par d'autres recherches plus étendues.

\subsection{Perception de la dangerosité des diverses sources de distraction}

Le tableau 4 présente les cotes moyennes de dangerosité perçue des diverses sources de distraction, calculées sur la base des cotes attribuées par l'ensemble des répondants à chacun des énoncés du questionnaire et ramenées sur 100.

Il y paraît clairement que les répondants différencient le niveau de dangerosité entre les diverses sources de distraction, les cotes globales attribuées aux items allant de 93,9 points, signifiant qu'il y a unanimité parmi les répondants sur le niveau élevé de dangerosité de l'item concerné, à 32,4 points, dénotant un large consensus parmi les répondants sur le niveau peu élevé de dangerosité de l'item concerné.

La cote clairement basse accordée par les répondants à la dangerosité de l'utilisation du téléphone portable à main libre révèle que cette fausse croyance est largement répandue même chez une population de niveau éducatif élevé comme celle concernée par cette étude.

Par ailleurs, dans $71 \%$ des cas les hommes jugent les situations de distraction moins dangereuses que les femmes et ceci vaut aussi bien pour les répondants qui ont eu des collisions dues à la distraction au volant que pour les répondants qui n'en ont pas eu. Dans la même proportion, les répondants qui ont eu des collisions dues à la distraction au volant jugent les diverses situations comme moins dangereuses que les répondants qui n'en ont pas eu et cela vaut pour les hommes comme pour les femmes.

\subsection{Comportements des répondants eu égard aux diverses sources de distraction}

Le tableau 5 présente les cotes moyennes représentant les déclarations des répondants quant à la fréquence de leurs 
Tableau 3 Moyennes d'âge des répondants ayant eu des collisions et pourcentages de ces répondants ayant 26 ans ou moins selon la source de distraction ayant causé la collision et le genre

\begin{tabular}{|c|c|c|c|c|}
\hline & \multicolumn{2}{|c|}{ Moyenne d'âge } & \multicolumn{2}{|c|}{$\begin{array}{l}\text { \% de répondants ayant } \\
26 \text { ans ou moins }\end{array}$} \\
\hline & $\mathbf{M}$ & $\mathbf{F}$ & $\mathbf{M}$ & $\mathbf{F}$ \\
\hline Répondants n'ayant pas eu des collisions & 43,8 & 36 & 15,4 & 28,5 \\
\hline Répondants ayant eu des collisions & 42,4 & 34,6 & 19 & 34 \\
\hline 1. Être absorbé par ses propres pensées & 44,3 & 32,8 & 12 & 43 \\
\hline 2a. Parler au téléphone portable & 43,8 & 37,3 & 13 & 43 \\
\hline 2b. Lire un message texte & 35 & 33,8 & 33 & 40 \\
\hline 2c. Écrire un message texte & 35,3 & 25,9 & 25 & 63 \\
\hline 3. Gérer une situation à l'intérieur du véhicule & 52,3 & 39,4 & 0 & 0 \\
\hline 4. Être distrait par le paysage ou un événement extérieur au véhicule & 39,9 & 34,3 & 14 & 33 \\
\hline 5. Etre distrait par la musique trop forte ou par la manipulation de la radio & 26 & 24,8 & 33 & 50 \\
\hline 6. Parler à un passager du véhicule & 28,4 & 25,5 & 60 & 50 \\
\hline 7. Manger et boire, avoir une activité reliée au tabac & 41,3 & 44,3 & 33 & 0 \\
\hline
\end{tabular}

comportements de distraction relatifs à chacun des énoncés du questionnaire, ramenées sur 100.

Les données de ce tableau révèlent que si les deux comportements que les répondants manifestent avec la plus haute fréquence peuvent être considérés comme relativement anodins (ajuster la climatisation et parler avec un passager, même si cette dernière source de distraction est apparue être impliquée dans les collisions rapportées par les répondants de cette étude dans 5,4\% des cas), les comportements qui suivent avec assez de fréquence (cotes moyennes allant de 1,2 à 1,5 sur une échelle allant de zéro à trois) comportent autant des distractions visuelles que des distractions cognitives jugées dangereuses, dont l'utilisation du téléphone portable.

Par ailleurs, dans tous les cas de figure, les femmes n'ayant pas eu des collisions manifestent moins de comportements de distraction que les femmes ayant eu des collisions, alors que, pour les hommes, c'est le cas pour $84,6 \%$ des items du questionnaire. De même, les femmes ayant eu des collisions manifestent des comportements de distraction plus que les hommes qui en ont eu dans $65,4 \%$ des cas.

\subsection{Corrélations entre la perception de dangerosité et les comportements rapportés}

L'examen de la corrélation entre les cotes attribuées à la dangerosité des diverses sources de distraction et les comportements personnels déclarés des répondants vis-à-vis ces mêmes sources montrent, comme il est permis de s'y attendre, une corrélation négative élevée se situant à $\mathrm{r}=-0,70(\mathrm{dl}=25 ; \mathrm{p}=0,001)$. L'examen des corrélations entre ces deux variables séparément pour les quatre sous-échantillons de l'étude montre que la corrélation la plus élevée concerne les hommes n'ayant pas eu de collisions dues à la distraction au volant $(\mathrm{r}=-0,77)$ alors que la corrélation la plus faible concerne les femmes qui ont eu de telles collisions $(\mathrm{r}=-0,61)$, les deux autres corrélations se situant près de la corrélation générale, soit $r=-0,68$ pour les femmes n'ayant pas eu de collision et $\mathrm{r}=-0,70$ pour les hommes qui en ont eu.

\subsection{Complémentarité entre le niveau perçu de dangerosité et le comportement}

Partant de l'hypothèse que si toute chose était égale par ailleurs, les cotes de dangerosité et les scores de comportement devraient être inversement proportionnels, c'est-à-dire totaliser 100 , un tel calcul a été réalisé pour l'ensemble des répondants et pour chacun des quatre sous-échantillons séparément.

Les données démontrent que, pour l'ensemble, cette hypothèse est quasi parfaitement appuyée. En effet, la moyenne générale des cotes et des scores jumelés s'établit à 99,3 avec certaines différences notables d'un item à l'autre du questionnaire. 
Tableau 4 Cotes moyennes attribuées par les répondants à la dangerosité de chacune des sources de distraction, par ordre descendant

\begin{tabular}{|c|c|c|c|c|c|c|}
\hline \multirow[b]{2}{*}{ No. } & \multirow[b]{2}{*}{ Énoncé } & \multicolumn{5}{|l|}{ Cote } \\
\hline & & $\begin{array}{l}\text { M ayant } \\
\text { eu } \\
\text { collision }\end{array}$ & $\begin{array}{l}\text { F ayant } \\
\text { eu } \\
\text { collision }\end{array}$ & $\begin{array}{l}\text { M } \\
\text { n'ayant } \\
\text { pas eu } \\
\text { collision }\end{array}$ & $\begin{array}{l}\text { F } \\
\text { n'ayant } \\
\text { pas eu } \\
\text { collision }\end{array}$ & Globale \\
\hline 1 & Écrire un message texte. & 94,1 & 92,3 & 95 & 94 & 93,9 \\
\hline 2 & Lire un journal, une carte ou tout autre matériel écrit. & 92,1 & 92,5 & 95,4 & 93,7 & 93,6 \\
\hline 3 & Écrire un mémo, une adresse, etc. & 88,3 & 85,7 & 92 & 91 & 90,2 \\
\hline 4 & Regarder en direction d'un passager assis sur la banquette arrière. & 86,4 & 86,7 & 85,5 & 87,9 & 87,1 \\
\hline 5 & $\begin{array}{l}\text { Vouloir contrôler un enfant, un animal ou un insecte dans le } \\
\text { véhicule. }\end{array}$ & 85,6 & 89,1 & 85,4 & 87,5 & 87,1 \\
\hline 6 & Lire un message texte. & 85,8 & 85,7 & 87 & 87,5 & 86,9 \\
\hline 7 & Entreprendre des soins corporels. & 83,5 & 84 & 86,1 & 83,7 & 84,5 \\
\hline 8 & Chercher à saisir un téléphone portable. & 79,9 & 81,7 & 81,9 & 84,8 & 83 \\
\hline 9 & Chercher à saisir un objet dans le véhicule. & 79,4 & 85,1 & 82,3 & 82,8 & 82,6 \\
\hline 10 & Ajuster l'équipement de navigation. & 76,1 & 78,7 & 74,5 & 74,5 & 75,1 \\
\hline 11 & Composer un numéro de téléphone. & 71,6 & 70,5 & 75,4 & 73,1 & 73,1 \\
\hline 12 & Regarder l'équipement de navigation. & 70,3 & 75,6 & 69,2 & 72,2 & 72 \\
\hline 13 & Avoir une conversation téléphonique l'appareil tenu en main. & 69,6 & 67,7 & 71,1 & 71,9 & 71,1 \\
\hline 14 & Être absorbé par ses propres pensées. & 67,1 & 67,4 & 63,4 & 70,4 & 68,2 \\
\hline 15 & Regarder en direction d'un passager assis sur le siège avant. & 60,6 & 65,6 & 61,3 & 66,2 & 64,2 \\
\hline 16 & $\begin{array}{l}\text { Regarder les accidents et autres événements survenant sur la } \\
\text { chaussée. }\end{array}$ & 58,7 & 62,4 & 59,5 & 64,6 & 62,3 \\
\hline 17 & Regarder les annonces publicitaires. & 53,8 & 60,9 & 57,3 & 64,3 & 60,7 \\
\hline 18 & Écouter la musique à volume élevé. & 61,2 & 55,9 & 60,6 & 58,6 & 59,5 \\
\hline 19 & $\begin{array}{l}\text { Ajuster le siège, les miroirs, le volant, les fenêtres, les fermetures } \\
\text { de porte, la ceinture de sécurité et autres. }\end{array}$ & 50 & 55,8 & 54 & 58,8 & 56 \\
\hline 20 & Regarder le paysage, les passants, etc. & 50 & 56,3 & 52,2 & 58,8 & 55,6 \\
\hline 21 & Fumer. & 47,5 & 54,4 & 52,7 & 58,7 & 55 \\
\hline 22 & Manger ou boire. & 55,4 & 48,9 & 56,3 & 52,8 & 53,8 \\
\hline 23 & Utiliser des cassettes, CDs et autres équipements de musique. & 44,6 & 47 & 42,4 & 43,5 & 43,7 \\
\hline 24 & Avoir une conversation téléphonique à main libre. & 32,5 & 35,8 & 35,4 & 38,3 & 37 \\
\hline 25 & Ajuster l'air climatisé, le chauffage, etc. & 35,7 & 37,9 & 33,5 & 36,9 & 35,7 \\
\hline 26 & Parler avec un passager. & 26,6 & 32,6 & 25,1 & 37,5 & 32,4 \\
\hline Total & & 65,6 & 67,5 & 66,7 & 69 & 67,9 \\
\hline
\end{tabular}

Les différences notables à la baisse concernent les sources de distraction suivantes : Fumer (moyenne globale de 69,3 points) ; Avoir une conversation par l'usage d'un téléphone portable à main libre (79,7 points) ; Manger ou boire (81,3 points) ; Écouter la musique à tue-tête $(82,0$ points). Il serait permis de croire que dans ces différents cas, le comportement ne constitue pas un complément parfait à la dangerosité perçue non en raison du niveau de dangerosité de ce comportement sur la sécurité routière mais pour d'autres raisons liées, par exemple, à l'abstention de l'acte de fumer en soi, à la non-disponibilité au conducteur d'un dispositif à main libre pour le téléphone portable, aux habitudes culturelles concernant le caractère répréhensible de manger ou boire au volant aux yeux des répondants dont les caractéristiques sociales sont particulières, ou à l'âge de la majorité des répondants qui ont dépassé la période où ils apprécieraient l'écoute de la musique à tue-tête.

En contrepartie, l'examen des cas où les cotes de dangerosité et les scores du comportement se démarquent de 
Tableau 5 Scores moyens déclarés par les répondants quant au comportement personnel eu égard à chacune des sources de distraction, par ordre descendant

\begin{tabular}{|c|c|c|c|c|c|c|}
\hline \multirow[b]{2}{*}{ No. } & \multirow[b]{2}{*}{ Énoncé } & \multicolumn{5}{|l|}{ Score } \\
\hline & & $\begin{array}{l}\text { M ayant eu } \\
\text { collision }\end{array}$ & $\begin{array}{l}\text { F ayant eu } \\
\text { collision }\end{array}$ & $\begin{array}{l}\text { M n'ayant } \\
\text { pas eu } \\
\text { collision }\end{array}$ & $\begin{array}{l}\text { F n'ayant } \\
\text { pas eu } \\
\text { collision }\end{array}$ & Global \\
\hline 1 & Ajuster l'air climatisé, le chauffage, etc. & 59,9 & 68,2 & 62,2 & 63,1 & 63,3 \\
\hline 2 & Parler avec un passager. & 66,2 & 59,8 & 65,2 & 54,6 & 61,5 \\
\hline 3 & Regarder le paysage, les passants, etc. & 53,6 & 46,6 & 47,6 & 41,1 & 47,2 \\
\hline 4 & Regarder les annonces publicitaires. & 53,6 & 46,1 & 46,3 & 42,3 & 47,1 \\
\hline 5 & Utiliser des cassettes, CDs et autres équipements de musique. & 45,7 & 48,8 & 46,8 & 45,2 & 46,6 \\
\hline 6 & Être absorbé par ses propres pensées. & 49,8 & 52,3 & 40 & 42,2 & 46,1 \\
\hline 7 & Avoir une conversation téléphonique à main libre. & 46 & 47,3 & 47,2 & 36,4 & 44,2 \\
\hline 8 & Avoir une conversation téléphonique l'appareil tenu en main. & 46 & 44,3 & 39,4 & 37,9 & 41,9 \\
\hline 9 & Composer un numéro de téléphone. & 44,9 & 45,5 & 39,5 & 36,6 & 41,6 \\
\hline 10 & Lire un message texte. & 39,2 & 46,7 & 34,2 & 33,5 & 38,4 \\
\hline 11 & $\begin{array}{l}\text { Regarder les accidents et autres événements survenant sur la } \\
\text { chaussée. }\end{array}$ & 38,4 & 33,3 & 35,4 & 29,9 & 34,3 \\
\hline 12 & Chercher à saisir un téléphone portable. & 32,5 & 42 & 30,1 & 29,5 & 33,5 \\
\hline 13 & Regarder en direction d'un passager assis sur le siège avant. & 36,3 & 33,7 & 32,3 & 27 & 32,3 \\
\hline 14 & $\begin{array}{l}\text { Ajuster le siège, les miroirs, le volant, les fenêtres, les fermetures } \\
\text { de porte, la ceinture de sécurité et autres. }\end{array}$ & 31,3 & 33,7 & 29,1 & 30,5 & 31,1 \\
\hline 15 & Écrire un message texte. & 28,3 & 37,3 & 24,5 & 26,3 & 29,1 \\
\hline 16 & Manger ou boire. & 29,5 & 31,8 & 24,1 & 26,2 & 27,9 \\
\hline 17 & Regarder l'équipement de navigation. & 28,4 & 29,4 & 31,3 & 19 & 27 \\
\hline 18 & Chercher à saisir un objet dans le véhicule. & 23,1 & 32,1 & 18,1 & 22,1 & 23,8 \\
\hline 19 & Écouter la musique à volume élevé. & 21,9 & 26,7 & 20,5 & 22,5 & 22,9 \\
\hline 20 & Ajuster l'équipement de navigation. & 23,4 & 26,5 & 22,3 & 17,7 & 22,5 \\
\hline 21 & Fumer. & 20,4 & 17,7 & 15,5 & 10,3 & 16 \\
\hline 22 & Écrire un mémo, une adresse, etc. & 19 & 17,3 & 15 & 11,3 & 15,7 \\
\hline 23 & Regarder en direction d'un passager assis sur la banquette arrière. & 11,7 & 16,9 & 11,5 & 11,3 & 12,8 \\
\hline 24 & $\begin{array}{l}\text { Vouloir contrôler un enfant, un animal ou un insecte dans le } \\
\text { véhicule. }\end{array}$ & 11,3 & 16,7 & 10,6 & 10,8 & 12,3 \\
\hline 25 & Lire un journal, une carte ou tout autre matériel écrit. & 7,1 & 10,5 & 6,2 & 6,5 & 7,6 \\
\hline 26 & Entreprendre des soins corporels. & 4 & 13,9 & 1,1 & 5,9 & 6,2 \\
\hline Total & & 33,5 & 35,6 & 30,6 & 28,5 & 32 \\
\hline
\end{tabular}

100 à la hausse et qui dénotent que malgré une perception donnée de la dangerosité de la source de distraction, les personnes manifestent le comportement concerné au-delà de l'attente normale, montre ce qui suit : des 48 cas relevés sur un total de 104 (26 items par 4 sous-échantillons), 11 items sur 26 totalisent plus de 100 points dont 9 totalisent plus de 100 points chez chacun des 4 sous-échantillons considérés, reflétant ainsi une tendance générale chez les répondants, indépendamment de leur position quant à l'occurrence de collisions dues à la distraction au volant. Ces items sont dans l'ordre : « Lire un message texte » (124,9 points) ; « Écrire un message texte » (122,9 points) ; « Chercher à saisir un téléphone portable » (115,6 points) ; «Composer un numéro de téléphone » (114,3 points) ; « Être absorbé par ses propres pensées » (113,1 points) ; "Avoir une conversation téléphonique l'appareil tenu à la main » $(112,0$ points) ; "Chercher à saisir un objet dans le véhicule » (106,2 points) ; « Regarder les annonces publicitaires » (106,1 points) ; et « Écrire un mémo, une adresse, etc. » (104,9 points). 
Les données révèlent par ailleurs que l'occurrence des dépassements du seuil de 100 points est plus fréquente chez les femmes qui ont eu des collisions dues à la distraction au volant. En effet, ce groupe de répondants totalise 16 items sur 26 où il dépasse 100 points, alors que les trois autres sous-échantillons se partagent également les autres occurrences. De même, sur ces 16 items, ce groupe se situe presque toujours en tête des trois autres groupes, avec variation entre 102,9 points pour « Écrire un mémo ou une adresse » et « Regarder le paysage », et un pic de 132,4 points pour «Lire un message texte » et 129,6 points pour «Écrire un message texte».

\section{Discussion}

L'occurrence de collisions dues à la distraction au volant paraît ne pas être un phénomène rare, même chez une population de niveau éducatif supérieur comme c'est le cas des sujets de cette étude dont $30 \%$ rapportent avoir eu de telles collisions. La grande majorité des sources de distraction évoquées par les répondants comme causes de ces collisions implique une distraction cognitive, comme c'est le cas de l'utilisation du téléphone portable et des rêves éveillés. Les autres sources de distraction visuelle, manuelle et auditive concernées comportent aussi, de toute évidence, une composante cognitive de détournement de l'attention vers une activité rivale à la tâche de conduite, comme c'est le cas de la gestion d'une situation à l'intérieur du véhicule, de la distraction par le paysage ou la musique et du fait de parler avec un passager du véhicule. Ces résultats confirment les recherches récentes sur les capacités limitées du cerveau à s'acquitter adéquatement de deux tâches à la fois [11].

Si l'on compare ces résultats avec ceux obtenus par la NHTSA sur un large échantillon de collisions qui ont eu lieu durant les années 2005, 2006 et 2007 [6], et que l'on considère uniquement les collisions où la distraction au volant est impliquée (45,1\% de l'ensemble des collisions considérées dans l'étude de la NHTSA), force est de constater certaines similitudes entre ces deux études, mais aussi deux différences notoires. En effet, si les événements internes à l'esprit de la personne occupent une place importante dans les distractions ayant causé des collisions (29,7 \%, deuxième cause), similaire à celle obtenue dans la présente étude, la première source de distraction présente dans les collisions étudiées par la NHTSA durant la période concernée se trouve être la conversation avec les passagers $(35,3 \%$ des collisions où la distraction au volant est en cause contre 5,4\% dans l'échantillon de la présente étude), alors que l'utilisation du téléphone portable n'a été identifiée que dans $7,5 \%$ des cas. Il est à noter cependant que les données de cette étude américaine concernent les collisions qui ont eu lieu il y a de huit à dix ans, donc, vraisemblablement avant que l'utilisation du téléphone portable ne soit aussi généralisée qu'aujourd'hui, surtout pour l'écriture de messages. En effet, les données relatives aux collisions fatales aux États-Unis en 2011 révèlent que $10 \%$ de ces collisions sont dues à la distraction au volant alors que $17 \%$ des collisions qui ont causé des dommages corporels sont dues à la distraction [12]. Et sur les causes connues de distraction, les principales concernent, en ordre descendant, « être absorbé par ses propres pensées » $(33,3 \%$, versus $29,5 \%$ dans la présente étude), « utiliser le téléphone portable » $(21 \%$, versus $29,5 \%)$, « être distrait par une personne, un objet ou un événement extérieur » $(10,7 \%$, versus $7,8 \%$ ) et «parler avec un passager ou le regarder » $(7,7 \%$, versus $5,4 \%)$, soit une différence notable en ce qui concerne l'usage du téléphone portable qui est plus présent dans les collisions survenues à la population de cette étude.

Quant aux autres sources de distraction, la première étude américaine montre des résultats étonnamment similaires à ceux de la présente étude pour ce qui est de la gestion d'objets et d'événements à l'intérieur du véhicule $(13,1 \%$ versus $13,2 \%)$ et de manger, boire et fumer $(4,9 \%$ versus $4,7 \%$ ), alors que la deuxième étude constate une baisse de l'occurrence de collisions dues à la gestion de situations à l'intérieur du véhicule (3,9\% des cas).

Par ailleurs, contrairement à la classification de Regan et al. [3] et de Regan et Strayer [4] qui mettent comme sources de distraction interne au véhicule, à la fois l'utilisation du téléphone portable et se préoccuper outre mesure des indications du tableau de bord, il parait judicieux de différencier ces deux types de sources de distraction comme il est proposé par Engstrom et al. [2]. En fait, les résultats de la présente étude confirment que ces deux types de distraction ont des effets différentiels sur l'incidence de collision : l'utilisation du téléphone portable, qui n'est d'aucune manière reliée à l'activité de conduite est susceptible de détourner l'attention du conducteur de manière dangereuse de cette activité, alors que la manipulation des équipements du véhicule, tel l'ajustement de la climatisation ou le maniement des fenêtres, qui se situe, selon Engstrom et al. [2, p. 17] au milieu de l'échelle de lien avec la tâche de conduite et qui relève, de toute évidence, des activités automatisées [15, p. 21], semble se passer sans danger chez les sujets de cette étude dont très peu sont des conducteurs novices.

L'effet âge est particulièrement perceptible autant chez les hommes que chez les femmes ayant eu des collisions en ce qui concerne les sources suivantes de distraction : écriture d'un message texte, écoute de la musique à tue-tête et manipulation de la radio et le fait de parler à un passager, pour lesquelles l'âge est significativement plus bas que celui de l'ensemble des répondants. S'ajoute à cela pour les hommes la lecture d'un message texte. Il y a aussi, parmi les répondantes ayant eu des collisions, 
une nette prédominance des moins de 26 ans par rapport à l'ensemble des répondantes. Ceci montre qu'il existe une forte incompatibilité entre être un conducteur relativement novice et se permettre les comportements de distraction susmentionnés. Ceci tend aussi à confirmer, chez les sujets concernés, le retard dans la maturation des fonctions cognitives supérieures de discernement, d'anticipation du danger et de contrôle des impulsions du moment [10], maturation qui commanderait à ces sujets de s'abstenir des comportements concernés et de centrer leur attention sur la tâche de conduite.

Par ailleurs, si l'on conçoit aisément que la lecture et l'écriture d'un message texte pendant la conduite d'un véhicule et l'écoute de la musique à tue-tête soient des phénomènes de jeunesse, on peut se demander pourquoi le fait de parler à un passager comporte un danger de collision chez les sujets les plus jeunes alors qu'il comporterait un danger moindre chez les sujets moins jeunes. On pourrait dès lors avancer l'hypothèse que les plus jeunes deviennent plus absorbés par l'interaction avec les autres passagers du véhicule de sorte à détourner leur attention des exigences de la conduite, s'exposant ainsi à plus de risque de collision. Il est aussi possible d'évoquer dans ce contexte l'influence que les pairs passagers du véhicule peuvent avoir sur le jeune conducteur, surtout de sexe masculin, ayant pour effet le détournement de son attention et les risques qui en résulteraient [16].

Quant à la perception de la dangerosité des sources de distraction, les résultats démontrent une différenciation claire entre celles-ci, allant généralement dans le sens des attentes normales à ce sujet. En effet, sept des 8 sources de distraction liées à l'utilisation du téléphone portable et à l'équipement de navigation se trouvent être parmi les 13 sources (sur 26) jugées les plus dangereuses. Les autres sources de distraction en tête de liste en termes de dangerosité concernent certaines activités similaires telles « lire un journal » ou «écrire un mémo » ou des activités requérant un détournement important de l'attention tels « regarder en direction d'un passager assis sur la banquette arrière », " vouloir contrôler un enfant ou un animal en mouvement » ou « entreprendre des soins corporels ». Le fait d'être absorbé par ses propres pensées arrive en quatorzième place de dangerosité à 68,2 points, alors qu'il occupe, avec l'utilisation du téléphone portable, la première place comme cause de collision. Les répondants semblent donc sous-estimer la dangerosité de cette source de distraction. Les répondants ont aussi tendance à sous-estimer d'autres sources de distraction telles que l'écoute de la musique à volume élevé et la manipulation des équipements de musique, manger et boire qui ont été mentionnées comme causes d'un pourcentage notable de collisions et, surtout, avoir une conversation téléphonique à main libre.
Compte tenu des résultats obtenus dans cette étude et de la position de la NHTSA à ce sujet, il y a lieu de se demander s'il ne faut pas classer « être absorbé par ses propres pensées » comme source de distraction interne au sujet comme le suggèrent Regan et al. [4] et Regan et Strayer [3] et la différencier des autres formes d'inattentions dont traitent, entre autres, Lemercier et al. [17] et Bailly et Chapon [18].

Si l'on compare les résultats concernant la perception de dangerosité de l'utilisation du téléphone portable aux résultats obtenus au Québec par la SAAQ [8], force est de constater que les résultats sont étonnamment similaires, les Québécois comme les Libanais étant pratiquement unanimes à juger le fait de lire ou d'écrire un message texte comme très dangereux $[9, \mathrm{p} .31]$ alors qu'utiliser le téléphone portable pour avoir une conversation est jugé plutôt dangereux et ce dans les mêmes proportions [9, p. 9]. Par contre, s'agissant de l'auto-rapport des comportements à risque des conducteurs liés à l'utilisation du téléphone portable [9, p. 33ss], force est de constater que les Libanais concernés par la présente étude rapportent utiliser le téléphone portable beaucoup plus fréquemment que les Québécois, soit près de deux fois plus pour le fait de parler au téléphone portable et quatre fois plus en ce qui concerne le fait de lire ou d'écrire un message texte.

L'inadéquation entre le niveau perçu de dangerosité de certaines sources de distraction et le comportement vis-à-vis de ces mêmes sources pose des questions sérieuses quant aux capacités humaines d'autorégulation du comportement. En particulier, le penchant à utiliser le téléphone portable alors que l'on conduit un véhicule en marche, malgré la conscience du danger d'un tel comportement, rappelle les recherches en santé qui démontrent, par exemple, que malgré la conscience du danger du tabagisme, un grand nombre de personnes persistent à fumer. En effet, n'est-il pas significatif que sur les 9 items du questionnaire où la somme de la cote de dangerosité et du score de comportement dépasse le seuil de 100 points figurent 5 des 6 items qui ont trait à l'utilisation du téléphone portable et se trouvent donc parmi les comportements les plus réfractaires à l'autorégulation?

En fait, il apparaît dans cette étude que la tentation d'utiliser le téléphone portable, d'une façon ou d'une autre, est plus forte que la conscience du danger que cela implique. Et il semble que l'individu doit avoir une volonté de fer pour résister à cette tentation; cela est particulièrement vrai pour les conductrices les plus jeunes. D'autres sources de distraction semblent aussi exercer une certaine attraction risquée sur les conducteurs, telles regarder les annonces publicitaires et se laisser absorber par ses propres pensées, et dénotent une faiblesse au niveau de l'auto-contrôle et de l'autorégulation du comportement de conduite automobile. Si l'on suit le raisonnement de Engstrom et al. [2, p. 21], 
l'attention dirigée serait une habileté qui s'acquiert à travers la répétition de la pratique avant de devenir automatisée. Ainsi, les conducteurs novices doivent déployer des efforts conscients et un contrôle cognitif dirigé pour déterminer sur quels aspects de la situation diriger leur attention.

Il y a lieu, enfin, de souligner la croyance erronée chez les sujets de cette étude, aussi éduqués soient-ils, à l'effet que l'utilisation du téléphone portable à main libre est relativement peu dangereuse, alors que la littérature scientifique et professionnelle dans ce domaine $[11,13,14]$ montre qu'une telle utilisation comporte des risques substantiels.

\section{Conclusion}

Les collisions dues à la distraction au volant sont des phénomènes relativement communs dans la vie de la population étudiée, qui ne diffère, à cet égard, que sur peu de points d'autres populations prises comme repères. Les sources les plus fréquentes de distraction ayant abouti à des collisions sont le fait d'être absorbé par ses propres pensées et l'utilisation du téléphone portable alors que le véhicule est en marche.

Il paraît difficile ou quasiment impossible que le conducteur d'un véhicule en marche évite en tout temps d'avoir des pensées positives ou négatives, anticipatives ou rétrospectives, émotives ou strictement cognitives, qui détournent son attention de la tâche de conduite vers ces pensées et préoccupations et réduisent ainsi sa vigilance vis-à-vis de ce qui se passe dans l'environnement de conduite menant à une absence d'attention ou à une attention insuffisante aux activités cruciales pour une conduite sécuritaire. Par contre, il est permis de croire qu'il est possible d'adopter diverses mesures pour contrer les autres sources de distraction au volant dont, en particulier, l'utilisation du téléphone portable pendant que le véhicule est en marche.

Ainsi, il y a lieu, de prime abord, d'intensifier les mesures de prévention primaire d'éducation et de sensibilisation des conducteurs aux dangers de la distraction au volant sous toutes ses formes. Il y a aussi lieu de déployer tous les efforts nécessaires en vue d'assurer un aménagement adéquat de l'environnement routier, en particulier la réduction des sources extérieures de distraction, et de rendre les routes plus « pardonnantes » pour ne pas faire subir au conducteur des conséquences graves pour la moindre distraction. Il faudrait, par ailleurs, prévoir qu'en cas de survenue de comportements distraits, les véhicules puissent avertir le conducteur des déviations critiques et des risques encourus et, comme les récents développements technologiques concernant les systèmes d'aide à la conduite automobile le laissent croire, qu'ils puissent être équipés de mécanismes de correction des erreurs du conducteur. A cela, peuvent s'ajouter des solutions technologiques pour bloquer la possibilité d'utilisation du téléphone portable pendant que le véhicule est en marche et réglementer ce comportement en appliquant des sanctions dissuasives à tout contrevenant.

Enfin, une attention particulière doit être portée aux mesures à prendre pour contrer le phénomène de l'utilisation du téléphone portable par les jeunes de moins de 26 ans qui courent des risques plus importants que leurs aînés. Il faudrait se demander s'il ne faut pas bannir complètement son utilisation au volant en bas de cet âge.

\section{Références}

1. Fort, A (2006) Les différents défauts de l'attention du conducteur : Distraction. In Défauts d'Attention et Conduite Automobile : état de l'art et nouvelles orientations pour la recherche dans les transports. Chapon, A., Gabaude, C. and Fort, A. (Eds). Les collections de l'Inrets - Synthèse Inrets $n^{\circ} 52$, Inrets, Paris, pp. $45-51$.

2. Engstrom, J, Monk, C, Hanowski, R, Horrey, W, Lee, J, McGehee, D, Regan, M, Stevens, A, Traube, E, Tuukanen, M, Victor, T, Yang, C (2013) A conceptual framework and taxonomy for understanding and categorizing driver inattention. EU-US ITS Cooperation. Brussels: European Commission. EU-US Inattention Taxonomy report Revised version September 2013-1.pdf (Consulté le 30/7/2015)

3. Regan, MA, Strayer, DL (2014) Towards an Understanding of Driver Inattention: Taxonomy and Theory. Annals of Advances in Automotive Medicine 58: 5-14.

4. Regan, ML, Hallett, C, Gordon, CP (2011) Driver distraction and driver inattention: Definition, relationship and taxonomy. Accident Analysis and Prevention 43: 1771-1781.

5. Chapon, A, Gabaude, C, Fort, A (Eds) (2006) Défauts d'Attention et Conduite Automobile : état de l'art et nouvelles orientations pour la recherche dans les transports. Les collections de l'Inrets Synthèse Inrets ${ }^{\circ} 52$, Inrets, Paris.

6. Singh, S (2010) Distracted Driving and Driver, Roadway, and Environmental Factors (Report No. DOT HS 811 380). National Highway Traffic Safety Administration, Washington, DC.

7. Schroeder, P, Meyers, M, Kostyniuk, L (2013) National survey on distracted driving attitudes and behaviors-2012 (Report No. DOT HS 811 729). National Highway Traffic Safety Administration, Washington, DC.

8. CAA-Québec (2007) La distraction au volant et ses impacts sur la conduite automobile. Enquête conjointe de CAA-Québec et de l'Équipe de l'émission JE (TVA), Québec, Canada.

9. SAAQ (2013) Sondage postcampagne et évaluation des comportements relatifs à la distraction au volant auprès de la population québécoise : Rapport d'analyse final. Société de l'assurance automobile - Léger Marketing, Québec, Canada.

10. Giedd, J (2009) The Teen Brain: Primed to Learn, Primed to Take Risks. The Dana Foundation (http://www.dana.org/Cerebrum/2009/The_Teen_Brain Primed_to_Learn,_Primed_to_Take_Risks/) (Consulté le 30 juillet 2015).

11. National Safety Council (2010) Understanding the Distracted brain: Why driving while using hands-free cell phones is risky behavior. NSC, Washington. 
12. NHTSA (2013) Distracted Driving 2011 (Report No. DOT HS 811 737). National Highway Traffic Safety Administration, Washington, DC.

13. Mondiale, Organisation de la Santé (2011) L'utilisation des téléphones mobiles : la distraction au volant, un problème qui s'aggrave. Organisation mondiale de la Santé, Genève, Suisse. (http://who.int/violence_injury_prevention/publications/road_ traffic/distracted_driving_fr.pdf?ua $=1 \& u a=1) \quad$ (Consulté le 30/7/2015)

14. Institut National de santé publique au Québec (2007) Avis de santé publique sur les effets du cellulaire au volant et recommandations. Institut national de santé publique, Québec, Canada.

15. Chapon, A, Bailly, B (2006) Attention et Conduite Automobile. In Défauts d'Attention et Conduite Automobile : état de l'art et nouvelles orientations pour la recherche dans les transports. Chapon, A., Gabaude, C. and Fort, A. (Eds). Les collections de 1'Inrets, Synthèse Inrets $\mathrm{n}^{\circ}$ 52, Inrets, Paris, pp. $18-22$.
16. Ouimet, MC, Simons-Morton, B, Zador, P, Lerner, N, Freedman, M, Duncan, G, Wang, J (2010) Using the US National Household Travel Survey to estimate the impact of passenger characteristics on young drivers' relative risk of fatal crash involvement. Accident Analysis and Prevention 42: 689-694.

17. Lemercier, C, Quaireau, C, Boujon, C, Chapon, A (2006) Les différents défauts de l'attention: Inattentions. In Défauts d'Attention et Conduite Automobile : état de l'art et nouvelles orientations pour la recherche dans les transports. Chapon, A., Gabaude, C. and Fort, A. (Eds). Les collections de l'Inrets, Synthèse Inrets $n^{\circ}$ 52, Inrets, Paris, pp. 51-55.

18. Bailly, B, Chapon, A (2006) Les troubles spécifiques de la sélectivité de l'attention: Conscience de la situation de conduite et attention du conducteur. In Défauts d'Attention et Conduite Automobile : état de l'art et nouvelles orientations pour la recherche dans les transports. Chapon, A., Gabaude, C. et Fort, A. (Eds). Les collections de L'Inrets, Synthèse Inrets $n^{\circ} 52$, Inrets, Paris, pp. 55-58. 